\title{
Self-assembled ErAs islands in GaAs for optical-heterodyne THz generation
}

\author{
C. Kadow, ${ }^{\text {a) }}$ A. W. Jackson, and A. C. Gossard \\ Materials Department, University of California-Santa Barbara, Santa Barbara, California 93106-5050 \\ S. Matsuura and G. A. Blake \\ Division of Geological and Planetary Science, California Institute of Technology MS150-21, Pasadena, \\ California 91125
}

(Received 9 February 2000; accepted for publication 17 April 2000)

\begin{abstract}
We report photomixer devices fabricated on a material consisting of self-assembled ErAs islands in GaAs, which is grown by molecular beam epitaxy. The devices perform comparably and provide an alternative to those made from low-temperature-grown GaAs. The photomixer's frequency response demonstrates that the material is a photoconductor with subpicosecond response time, in agreement with time-resolved differential reflectance measurements. The material also provides the other needed properties such as high photocarrier mobility and high breakdown field, which exceeds 2 $\times 10^{5} \mathrm{~V} / \mathrm{cm}$. The maximum output power before device failure at frequencies of $1 \mathrm{THz}$ was of order $0.1 \mu \mathrm{W}$. This material has the potential to allow engineering of key photomixer properties such as the response time and dark resistance. (C) 2000 American Institute of Physics.
\end{abstract}

[S0003-6951(00)01924-0]

A promising approach for the generation of submillimeter $(\mathrm{THz})$ waves is optical-heterodyne conversion, or photomixing, by photoconductive materials with response times in the picosecond (ps) range. ${ }^{1}$ The response time is a crucial parameter for device design and performance, and engineering control over this time is desirable. Brown et al. ${ }^{2}$ demonstrated photomixer devices based on low-temperature-grown (LTG) GaAs, which is the most widely used ultrafast photoconductor. $^{3-5}$

Here we report on the performance of photomixers made from an alternative material consisting of layers of ErAs islands separated by GaAs, as shown schematically in part (a) of Fig. 1. The use of this material was motivated by the recent finding that carrier trapping in GaAs:ErAs occurs on a subps time scale, similar to LTG GaAs. ${ }^{6}$ We believe that this material is particularly promising because one can engineer its microstructure, which in turn controls the electronic properties. The superlattice period, for example, controls the carrier trapping time. ${ }^{6}$ With molecular beam epitaxy $(\mathrm{MBE})$ it is straightforward to control this period and hence to achieve a desired carrier trapping time. Other properties that strongly depend on the microstructure are the dark resistance and the thermal conductivity.

The material used for the photomixer devices in the present work is grown by MBE on a semi-insulating (100) GaAs substrate. 60 periods of 1.2 monolayers of ErAs and $20 \mathrm{~nm}$ of GaAs are deposited on the substrate at a temperature of $535^{\circ} \mathrm{C}$, resulting in a $1.2 \mu \mathrm{m}$ thick film. The material is nominally identical to one of the samples discussed by Kadow et al., ${ }^{6}$ who also present details of the MBE growth. The response time measured by time-resolved differential optical reflectance for this material is $120 \mathrm{fs}$. The island di-

\footnotetext{
a) Author to whom correspondence should be addressed; electronic mail: kadow@engineering.ucsb.edu
}

ameters are between 1 and $2 \mathrm{~nm}$, and the density is about $7 \times 10^{12} \mathrm{~cm}^{-2}$.

Interdigitated electrodes with a finger width of $0.2 \mu \mathrm{m}$ and a finger spacing of $1.8 \mu \mathrm{m}$ were fabricated on the material surface. These electrodes are located at the vertex of a planar self-complementary log-spiral antenna. The interdigitated electrodes and the inner part of the spiral antenna are defined by electron beam lithography, the outer part of the spiral antenna by optical lithography. The metalization consists of $140 \mathrm{~nm}$ of gold on $40 \mathrm{~nm}$ of titanium. One of the devices is shown in part (b) of Fig. 1. We tested five devices from the same wafer. There is little scatter among the data from different devices.

The experimental setup is schematically shown in Fig. 2.

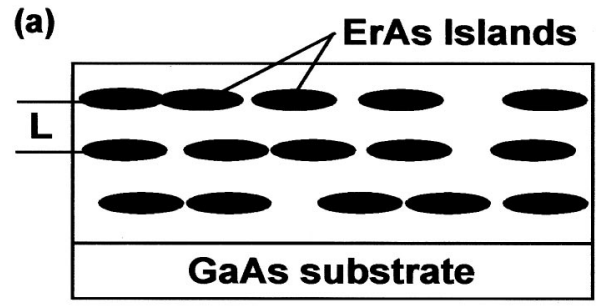

(b)

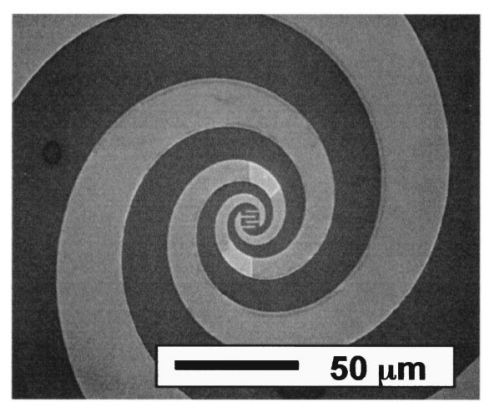

FIG. 1. (a) Schematic cross section of the samples showing the superlattice structure. (b) Top view of the metalization of the photomixer device. Shown are the interdigitated electrodes at the vertex of the spiral antenna. 


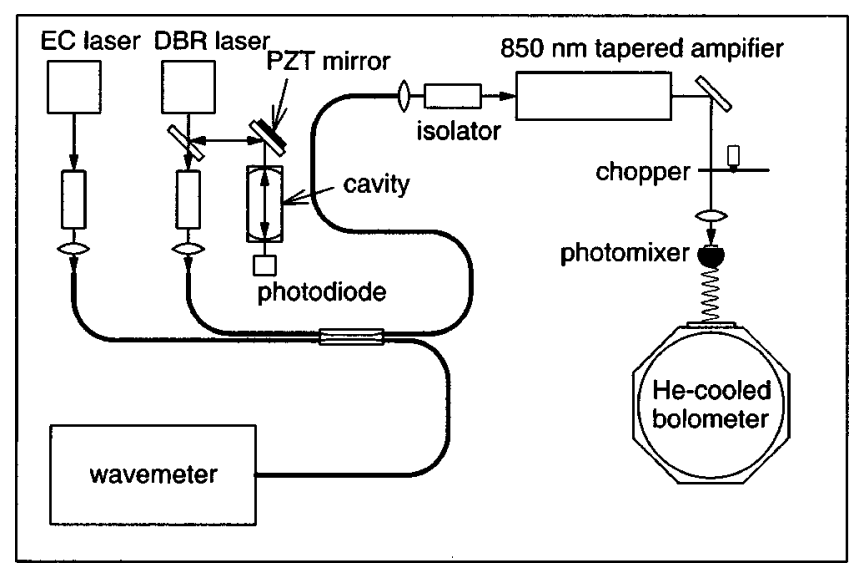

FIG. 2. Experimental photomixer test setup. Difference frequencies of over $10 \mathrm{THz}$ are possible with this system at a maximum power level of $500 \mathrm{~mW}$.

Two single-frequency diode lasers operating near $850 \mathrm{~nm}$ are used. The first is a grating-tuned external cavity laser, the second is a nearly fixed wavelength distributed Bragg reflector (DBR) laser locked to an external cavity. Both lasers emit approximately $30 \mathrm{~mW}$. The light from both laser diodes is coupled into individual single-mode optical fibers and combined in a 50-50 fiber coupler. Half of the power is coupled into a wave meter where the difference frequency $f$ is determined. The other half is outcoupled and amplified in an 850 $\mathrm{nm}$ tapered semiconductor optical amplifier. The maximum available power after amplification is $500 \mathrm{~mW}$. This signal is focused onto the interdigitated finger region of the photomixer devices. The focused spot is measured to be $6 \mu \mathrm{m}$ in diameter. We apply a dc bias $U_{\mathrm{DC}}$ to the interdigitated fingers and measure the dc photocurrent $I_{\mathrm{DC}}$. The submillimeter waves are predominantly emitted through the substrate and subsequently collimated with a hyperhemispherical $\mathrm{Si}$ lens. The output power is directed onto a Si bolometer, which is operated at liquid He temperature. We denote the detected power as $P_{\text {out }}$. The units are the rms voltage on the detector. From thermal blackbody calibrations, $650 \mathrm{mV} \mathrm{rms}$ corresponds to a power level of approximately $100 \mathrm{nW}$.

In the dark, the current-voltage $(I-V)$ characteristic of the devices is linear for biases below $2 \mathrm{~V}$. At higher bias the current increases superlinearly. The dark resistance in the linear region is $5 \mathrm{M} \Omega$. In the nonlinear region the data follow a power law with exponent 1.6. We expect the conductivity of the material to be strongly anisotropic due to its layered nature. We are currently not in a position to explain the details of the transport from a microscopic point of view. The exact behavior of the metal contacts is another unknown in these devices. Schmidt et al. ${ }^{7,8}$ performed transport measurements on similar samples with ohmic contacts to all ErAs containing layers to investigate the in-plane component of the dark conductivity. They find that the transport is thermally activated and strongly dependent on growth conditions. Different growth temperatures, for example, result in changes of several orders of magnitude in the sheet resistance. This can be understood in qualitative terms if the inplane transport is dominated by hopping between the ErAs islands, resulting in a strong dependence on island separation. From the $I-V$ measurements we also know that the breakdown field exceeds $2 \times 10^{5} \mathrm{~V} / \mathrm{cm}$. breakdown field exceeds $2 \times 10^{5} \mathrm{~V} / \mathrm{cm}$.
Downloaded 08 Mar 2006 to 131.215 .225 .174 . Redistribution subject to AlP license or copyright, see http://apl.aip.org/apl/copyright.jsp

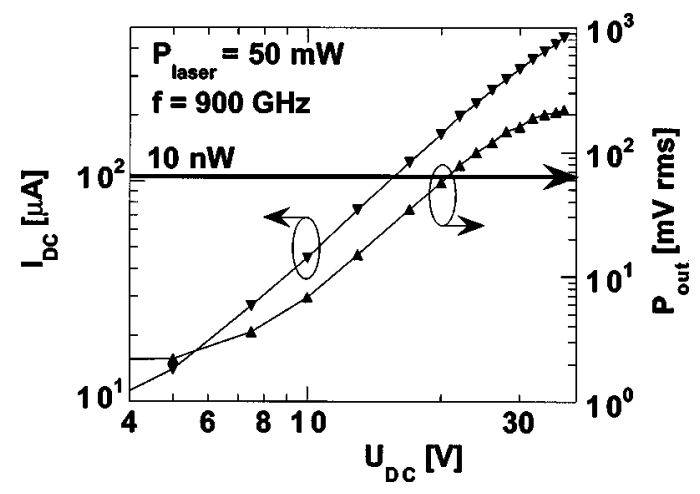

FIG. 3. Photocurrent $I_{\mathrm{DC}}$ and output power $P_{\text {out }}$ as a function of dc bias $U_{\mathrm{DC}}$ for one of the devices investigated. The laser power is $50 \mathrm{~mW}$ and the difference frequency is $900 \mathrm{GHz}$.

Figure 3 shows the dc photocurrent $I_{\mathrm{DC}}$ and the $\mathrm{THz}$ output power $P_{\text {out }}$ at a frequency $f$ of $0.9 \mathrm{THz}$ as a function of bias $U_{\mathrm{DC}} \cdot I_{\mathrm{DC}}$ increases superlinearly if $U_{\mathrm{DC}}$ is smaller than $25 \mathrm{~V}$, following a power law dependence with an exponent of approximately 1.7. Within our experimental error, this is the same exponent as for the dark currrent. The conduction mechanisms for dark and photocurrent are, however, not necessarily identical. At higher bias $\left(U_{\mathrm{DC}}>25 \mathrm{~V}\right)$ the dc photocurrent saturates. The output power $P_{\text {out }}$ increases in a monotonic fashion with applied bias. In an intermediate bias regime it appears to follow a power law dependence with an exponent around 3.0. At high bias, roughly at values larger than $25 \mathrm{~V}$, the output power saturates. Similar behavior is seen for devices made from LTG GaAs. Superlinear $I-V$ characteristics have been described by Brown et al. ${ }^{9}$ One explanation for the superlinear $I-V$ curve involves space charge limited currents. ${ }^{9}$ Another possible explanation is an electric field dependent carrier lifetime $\tau$ as observed by Zamdmer et al. in LTG GaAs. ${ }^{10}$ Saturation of the photocurrent has not been observed in LTG GaAs. The reason for the saturation behavior reported in this letter is not understood. Saturation of the output power has been observed by Jackson ${ }^{11}$ at similar THz frequencies. Under illumination the dc photocurrent increases linearly with the optical power. At $30 \mathrm{~V}$ bias the slope is $7 \times 10^{-3} \mathrm{~A} / \mathrm{W}$, which corresponds to an external quantum efficiency of $1 \%$. A small external quantum efficiency is expected from the short carrier lifetime. The value is similar to those measured on LTG GaAs. ${ }^{11}$ Some improvement of the external quantum efficiency could be achieved by incorporating an antireflection coating and by either increasing the thickness of the absorbing layer or incorporation of a DBR beneath the active region. ${ }^{11}$

The output power $P_{\text {out }}$ is expected to increase quadratically with optical power for heterodyne mixing. Fitting the data with $P_{\text {out }}=A P_{\text {laser }}^{2}$ yields $A=8 \times 10^{-6}$ at $f=946 \mathrm{GHz}$. When fitted with a power law, a slightly better fit is achieved with an exponent of 1.8. Figure 4 shows the frequency response from one device biased at $30 \mathrm{~V}$ for two different laser powers. The lower laser power of $50 \mathrm{~mW}$ results in a dc photocurrent of $340 \mu \mathrm{A}$, while the higher laser power of 90 $\mathrm{mW}$ leads to a dc photocurrent of $680 \mu \mathrm{A}$. A frequency corner can clearly be seen around $1.7 \mathrm{THz}$ in the data. At lower frequencies the power decreases by $3 \mathrm{~dB} /$ octave, but at 


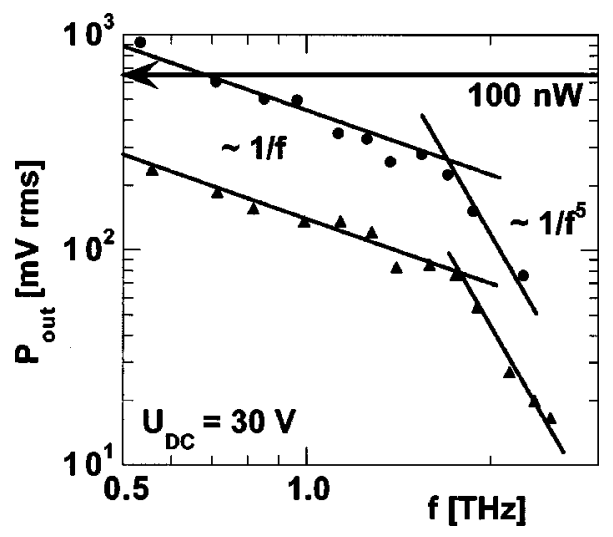

FIG. 4. Frequency response of one photomixer device biased at $30 \mathrm{~V}$ for two different laser powers. Circular symbols are for $P_{\text {laser }}=90 \mathrm{~mW}$, triangular symbols are for $P_{\text {laser }}=50 \mathrm{~mW}$.

octave. The latter number is only an estimate because the decay could only be observed over one octave as the power levels approach the thermal background. These two slopes are indicated in Fig. 4.

Brown et al. introduced a model for the frequency response of this type of device. ${ }^{12}$ The model contains two corner frequencies $f_{1}$ and $f_{2} . f_{1}$ is due to the $R C$ time constant of the electrical circuit $\left(f_{1}=1 / 2 \pi R C\right)$, and $f_{2}$ originates from the carrier lifetime in the photoconductor $\left(f_{2}\right.$ $=1 / 2 \pi \tau)$. The frequency dependence of our devices can be fit with this model if we assume the following: First, that the two frequencies $f_{1}$ and $f_{2}$ are equal $(\tau=R C)$ resulting in the corner at $1.7 \mathrm{THz}$ and second, the existence of an additional decay with $1 / f$ throughout the frequency range investigated. This modifies the reduced form of Eq. (9) in Ref. 12 to

$$
P_{f}=\frac{P_{0}}{f \cdot\left[1+(2 \pi \tau \cdot f)^{2}\right]^{2}} .
$$

The corner at $1.7 \mathrm{THz}$ gives a carrier lifetime of $100 \mathrm{fs}$, which is in good agreement with the 120 fs measured by pump-probe techniques. ${ }^{6}$ The radiation resistance of the antenna structure is $72 \Omega$. This yields a capacitance of $1.3 \mathrm{fF}$ for the observed corner, close to the capacitance of $1.6 \mathrm{fF}$ expected from calculations. The origin of the additional $1 / f$ rolloff is currently not understood. It may be related to the fact that the pump-probe data from nominally identical samples do not show a simple exponential decay, ${ }^{6}$ but contain a secondary decay, which indicates that a fraction of the carriers need longer than 120 fs to relax to their original state. These carriers may degrade the frequency response and lead to the $1 / f$ decay.
The maximum output power of the tested devices was limited by device failure as in the case for photomixers made from LTG GaAs. Each of the five tested devices was driven toward failure by increasing the laser power while keeping dc bias and frequency constant. The dc bias ranged between 25 and $30 \mathrm{~V}$, and the frequencies $f$ were between 0.9 and 1 THz. In order to compare the results, we scale the output power with the known frequency response to $1 \mathrm{THz}$. At 1 $\mathrm{THz}$ the average maximum output power was $85 \mathrm{nW}$, with a minimum of $55 \mathrm{nW}$ and a maximum of $106 \mathrm{nW}$. The laser power at failure was roughly $100 \mathrm{~mW}$, the dc photocurrent between 0.6 and $0.7 \mathrm{~mA}$, and the dissipated ohmic power about $17 \mathrm{~mW}$.

In conclusion, we have demonstrated that self-assembled ErAs islands in GaAs can be used to fabricate photomixer devices. The photomixer's frequency response demonstrates that the material is a photoconductor with subpicosecond response time, in agreement with time resolved differential reflectance measurements. It also provides the other needed properties such as high photocarrier mobility and high breakdown field. The breakdown field exceeds $2 \times 10^{5} \mathrm{~V} / \mathrm{cm}$. The maximum output power before device failure at frequencies of $1 \mathrm{THz}$ was of order $0.1 \mu \mathrm{W}$. The ability to reliably engineer important properties of the ErAs based material, for example the response time and the dark resistance, should result in devices with improved performance.

This research was supported by Jet Propulsion Lab under Contract No. 960776.

\footnotetext{
${ }^{1}$ For a review, see for example: S. Verghese, K. A. McIntosh, and E. R. Brown, IEEE Trans. Microwave Theory Tech. 45, 1301 (1997).

${ }^{2}$ E. R. Brown, K. A. McIntosh, K. B. Nichols, M. J. Manfra, and C. L. Dennis, Proc. SPIE 2145, 200 (1994).

${ }^{3}$ S. Matsuura, M. Tani, and K. Sakai, Appl. Phys. Lett. 70, 559 (1997).

${ }^{4}$ S. Verghese, K. A. McIntosh, and E. R. Brown, Appl. Phys. Lett. 71, 2743 (1997).

${ }^{5}$ S. Matsuura, G. A. Blake, R. A. Wyss, J. C. Pearson, C. Kadow, A. W. Jackson, and A. C. Gossard, Appl. Phys. Lett. 74, 2872 (1999).

${ }^{6}$ C. Kadow, S. B. Fleischer, J. P. Ibbetson, J. E. Bowers, A. C. Gossard, J. W. Dong, and C. J. Palmstrøm, Appl. Phys. Lett. 75, 3548 (1999).

${ }^{7}$ D. R. Schmidt, J. P. Ibbetson, D. E. Brehmer, C. J. Palmstrøm, and S. J. Allen, Mater. Res. Soc. Symp. Proc. 475, 251 (1997).

${ }^{8}$ D. R. Schmidt, A. G. Petukhov, M. Foygel, J. P. Ibbetson, and S. J. Allen, Phys. Rev. Lett. 82, 823 (1999).

${ }^{9}$ E. R. Brown, K. A. McIntosh, F. W. Smith, K. B. Nichols, M. J. Manfra, C. L. Dennis, and J. P. Mattia, Appl. Phys. Lett. 64, 3311 (1994).

${ }^{10}$ N. Zamdmer, Q. Hu, K. A. McIntosh, and S. Verghese, Appl. Phys. Lett. 75, 2313 (1999).

${ }^{11}$ A. W. Jackson, Ph.D. thesis, University of California at Santa Barbara, 1999.

${ }^{12}$ E. R. Brown, F. W. Smith, and K. A. McIntosh, J. Appl. Phys. 73, 1480
} (1993). 